\title{
Analysis of AODV Protocol against Pause Time Using NS2.34
}

\author{
Samiksha Nikam ${ }^{\mathrm{a}}$, B.T. Jadhav ${ }^{\mathrm{b}}$ \\ ${ }^{a}$ College ofConputer Application for Women's Satara, Affiliated to S.N.D.T. Women's University, Mumbai, \\ Santacruz(w) \\ ${ }^{b}$ Y.C. Institute of sciences, Satara, Affiliated to Shivaji University, Kolhapur
}

\begin{abstract}
An ad hoc network is very popular because of its easy deployment. It is a self-organising network which has dynamic topology. The routing is a major issue in the ad hoc network due to its node mobility. As nodes are moving continuously the source to destination pair is completely broken and repeated route discovery increases delay and network load and reduces throughput of ad hoc network. The routing protocols play a vital role in data transmission and affect the efficiency of the ad hoc network. Also, mobility factor affects the efficiency of the ad hoc network. The ad hoc network is said to be efficient if it transfers data with higher throughput, minimum delay and low overheads. In this paper, researcher studies efficiency of AODV protocol against node mobility. The node mobility term relates to the pause time i.e. the amount of time node is stable at a particular location. The high pause time means nodes in the network have low mobility and low pause time means nodes have high mobility. The performance of AODV protocol is evaluated on the basis of four performance matrices i.e. throughput, delay, routing load, and packet delivery ratio using the Network simulator NS2.34. This study will help the researcher further to regulate the scenario parameters of ad hoc network which will help to improve performance of the protocol.
\end{abstract}

Index Terms: AODV, Performance, network simulator, pause time.

(C) 2016 Published by MECS Publisher. Selection and/or peer review under responsibility of the Research Association of Modern Education and Computer Science

\section{Introduction}

Mobile ad hoc network is an autonomous collection of mobile users communicating over relatively constrained bandwidth. The network topology may change unpredictably and rapidly over the time. The main goal of an ad hoc network routing protocol is to establish an optimal route between source and destination node. The route should be discovered and maintained with a minimum overhead and bandwidth consumption. Routing is a key factor for transfer of packets from source to destination. [3]. Node mobility is an important parameter in ad hoc network which decides efficiency of the network. The efficiency of the network highly

* Corresponding author.

E-mail address: 
depends on the performance of the protocol. Due to mobile nodes topology of the network changes frequently and accordingly protocol has to update their routing tables. This might lead to increase routing load in the network. Thus, dynamic topology is one of the greatest challenges in the ad hoc network.

AODV protocol is popular on-demand routing protocol. It comprises new routing concept compared to DSDV routing protocol. It provides a scalable solution to relatively large network topology. In AODV protocol routes are created when source node want to transfer the data. Due to mobile nodes, sometimes nodes may not available to form a route. In such case delay and routing load increases as multiple route request packets flooded in the network. These also affect throughput and PDF of the network. It seems that mobility plays the vital role to decide performance of the protocol.

Many researchers have analysed the performance of AODV protocol by using different performance parameters under different circumstances. The main objective of this paper is to evaluate AODV protocol performance on the basis of varying pause time. Low pause time means high mobility and high pause time mean low mobility. Under predefined scenario and constrained like 50 mobile nodes, simulation area 500*500, maximum connections 10,512 bytes packet size, having fix mobility of $10 \mathrm{~m} / \mathrm{s}$ with varying pause time from 0 to $100 \mathrm{~ms}$. Throughput, delay, routing load and packet delivery fraction are the network performance parameters selected to investigate the performance of AODV protocol. A simulation study is carried out using network simulator ns2.34 [9].

This paper is organized as follows. The second section explains the working of AODV protocol and the performance parameters selected for evaluation; the third section describes the simulation environment and experimental performance data against pause time; the fourth section evaluates the efficiency of AODV protocol against node mobility and then we concluded at the end.

\section{Ad Hoc on Demand-Vector Routing Protocol (AODV) Protocol}

AODV routing protocol uses an on-demand approach for finding routes. It is packet routing protocol designed for use in mobile ad hoc network. It is based on DSDV and DSR algorithm. AODV protocol uses request/reply query approach for finding the route. Whenever there is a need for a path from any source to destination and if the route is not available then source initiate request packet (RREQ) containing destination address across the network [6,7]. Nodes receiving this packet update their information for the source node and set up backward pointers towards the source node in the routing tables. A node receiving the RREQ send a packet (RREP) as an acknowledgment, if it is either the destination or if it has a route to the destination. Source node receives RREP packet then route is established which starts data transmission. In reactive routing protocols, the route is calculated only when a node needs to send data to the destination node. Thus, route discovery is initiated only when needed. This saves overhead in maintaining unused routes. However, this may lead to larger initial delays [8].

\section{Methodology}

In this section researchers describe the performance metric, simulation process and environment, and experimental setup used to evaluate efficiency of AODV protocol.

\subsection{Performance Metrics}

The performance measurement of any network is an essential task because it helps to determine network performance need any upgrading for better performance. Basically three techniques are used to measure the performance of the network.

- Empirical Measurements

- Analytical Measurements 
- Simulation Measurements.

Once the system is built and it is in running phase empirical technique is used. During design and developing phase analytical and simulation technique is used. Researchers used simulation technique to measure the performance of AODV protocol. Routing protocol plays a vital role in data transmission in mobile ad hoc network. The protocol is said to be efficient if it is able to transfer data with low delay, high throughput, low routing load and higher packet delivery fraction. Following performance metrics have been selected to evaluate protocol performance [1].

- Throughput: It is measured as total number of packets successfully transmitted to the destination per unit time. It is measured in bits/sec. Throughput is calculated by using following formula,

Throughput $(\mathrm{bits} / \mathrm{sec})=($ Number of delivered packets $*$ Packet size $* 8) /$ Total simulation period

- Delay: it is measured as a total time required to transfer packets from source to destination. It also includes the delay caused by route discovery process and the queue in data packet transmission. Only the data packets that successfully delivered to the destinations that are counted. The lower delay in the network associated to better performance of the protocol.

- Packet delivery fraction (PDF): the ratio of the total number of generated packets to the number of delivered data packet to the destination. This illustrates the level of delivered data to the destination. The greater value of packet delivery ratio means the better performance of the protocol.

- Normalized Routing Load (NRL): it is measured as the number of routing packets transmitted per data packet delivered at the destination. Low routing load is desirable in the network which corresponds to better performance of the protocol

\subsection{Simulation process}

The simulations were performed using Network Simulator NS2.34. Initially scenario and traffic files are generated. These files are used as input for TCL script. After execution of TCL script two files are created i.e. NAM file and trace file. Trace files are used to analyze the behavior of network. Trace files are analyzed using AWK scripts. Ad hoc networks are highly dynamic hence simulation technique is an option to measure the performance. Table 1 shows list of simulation parameters and their values to run the simulation.

Following steps are performed to run the simulation.

- $\quad$ Select performance parameters. (Throughput, delay, routing load and packet delivery fraction).

- Generate scenario and topology files using cbrgen and setdest commands.

- Write TCL script (.tcl Extension file)

- Execute TCL script (Use ns Command)

- Generate Trace and NAM file.

- Execute AWK script to measure performance. 
Table 1. Simulation Environment

\begin{tabular}{ll}
\hline Parameter & Value \\
\hline Ad-hoc Routing Protocol & AODV \\
Antenna Type & Omni-directional \\
Simulation Time & $100 \mathrm{sec}$ \\
Simulation Area & 500 X 500 \\
Traffic Type & CBR \\
Node Speed & $10 \mathrm{~m} / \mathrm{s}$ \\
Data Packets & $512 \mathrm{bytes}$ \\
Pause Time & $0-100 \mathrm{~ms}$ \\
Number of Nodes & 50 \\
Mobility Model & Random Waypoint \\
Propagation Model & Two-ray Ground reflection \\
Interface Queue Type & Drop Tail/ Priority Queue \\
Interface Queue Length & 50 Packets \\
Max. Number of Connections & 10 \\
\hline
\end{tabular}

\subsection{Experiment Setup}

The goal of our experiments is to examine and compute performance of ad hoc network when AODV routing protocol is used. Each run of the simulator accept scenario file as input. The scenario file describe exact motion of each node and sequence of packets originated by each node together with exact time at which change in packet or motion occurs. To evaluate performance of ad hoc network we consider 10 random simulation runs to generate 10 random scenario patterns. The result is calculated by taking average of those 10 outputs. Experiments are carried out in two ways. Total 21 Simulations run each for $100 \mathrm{sec}$ and result is stored in Table 2. The fig1 shows screenshots of scenario generated for 50 nodes, pause time $25 \mathrm{~ms}$ and node speed $10 \mathrm{~m} /$ during simulation. Fig 2 depicts topology created for 50 nodes with cbr generator. Fig 3 shows screen shots of animation file while executing 50 nodes.

Table 2. Experimental Performance Data

\begin{tabular}{|c|c|c|c|c|c|}
\hline \multicolumn{6}{|c|}{ Experimental Design } \\
\hline & Scenario Parameter & & Perforn & ers & \\
\hline Sr. No & Pause Time & Throughput & Delay & PDF & NRL \\
\hline 1 & 0 & 48.27 & 87.498 & 98.92 & 1.203 \\
\hline 2 & 5 & 48.08 & 31.49 & 98.77 & 1.046 \\
\hline 3 & 10 & 47.82 & 80.005 & 98.15 & 1.466 \\
\hline 4 & 15 & 48.18 & 63.80 & 98.87 & 1.170 \\
\hline 5 & 20 & 48.12 & 49.982 & 98.58 & 1.525 \\
\hline 6 & 25 & 48.17 & 54.30 & 99.17 & 1.160 \\
\hline 7 & 30 & 48.21 & 55.521 & 98.85 & 1.279 \\
\hline 8 & 35 & 48.52 & 25.71 & 99.56 & 0.956 \\
\hline 9 & 40 & 48.58 & 52.44 & 99.03 & 1.195 \\
\hline 10 & 45 & 48.46 & 26.15 & 99.56 & 0.780 \\
\hline 11 & 50 & 46.56 & 49.446 & 95.33 & 3.502 \\
\hline 12 & 55 & 48.39 & 19.67 & 99.51 & 0.788 \\
\hline 13 & 60 & 48.34 & 40.807 & 99.15 & 1.076 \\
\hline 14 & 65 & 48.93 & 22.42 & 99.61 & 0.669 \\
\hline 15 & 70 & 48.78 & 58.805 & 89.52 & 0.814 \\
\hline 16 & 75 & 47.28 & 45.51 & 99.05 & 0.791 \\
\hline 17 & 80 & 48.65 & 33.061 & 99.24 & 0.772 \\
\hline 18 & 85 & 48.45 & 22.58 & 99.83 & 0.534 \\
\hline 19 & 90 & 48.55 & 41.572 & 89.76 & 0.605 \\
\hline 20 & 95 & 48.71 & 26.65 & 99.88 & 0.501 \\
\hline 21 & 100 & 48.85 & 46.666 & 99.92 & 0.514 \\
\hline
\end{tabular}




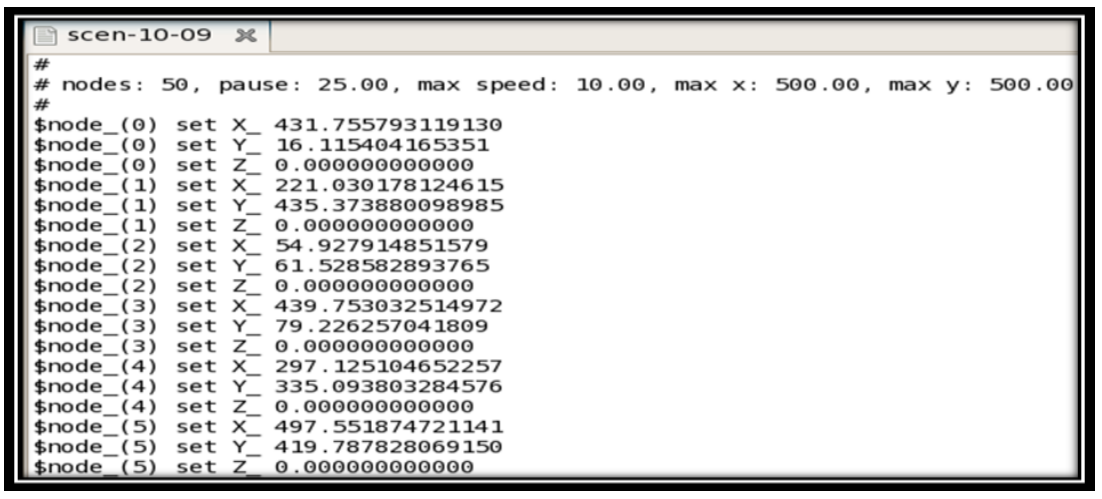

Fig.1. Screenshot of Scenario File Created using setdst Command

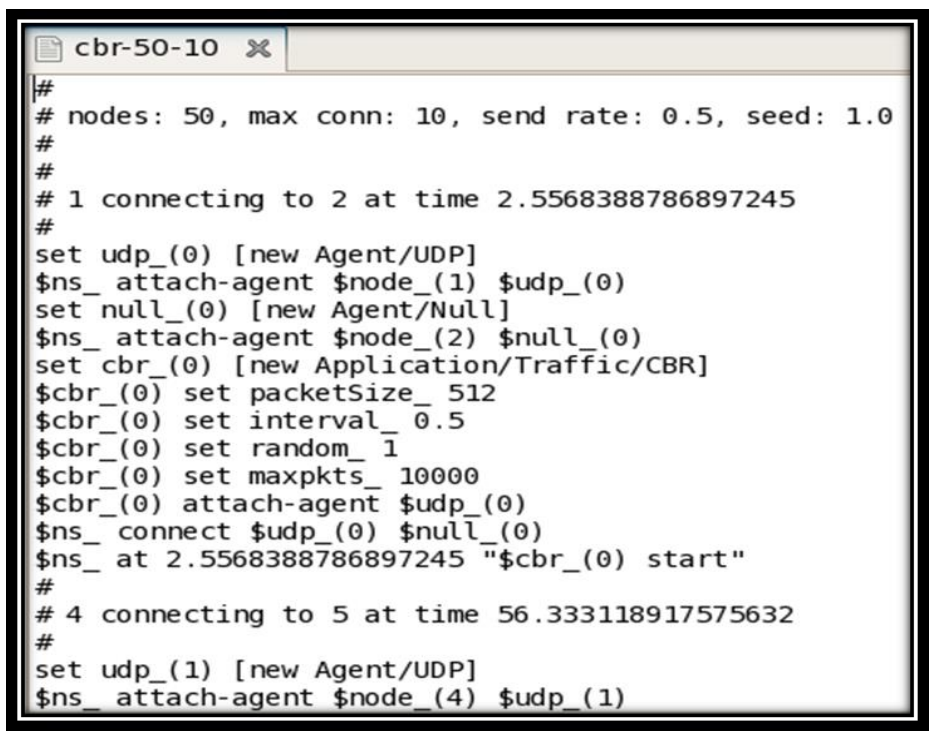

Fig.2. Screenshot of Topology Created using cbrgen Command
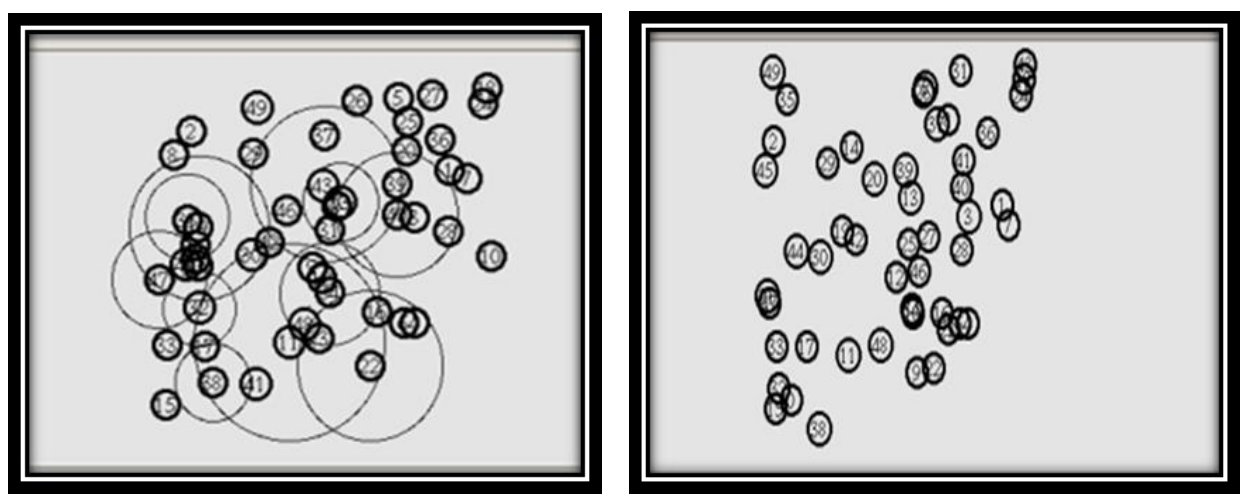

Fig.3. Screenshots of NAM File for Execution for 50 nodes 


\section{Performance Analysis}

Performance evaluation of AODV protocol with respect to pause time is mentioned below with graphical representation.

\subsection{Pause time vs. Throughput}

Graphical representation of throughput vs. pause time is shown in fig.4. It is observed that as pause time is increasing throughput is slightly increasing. Increasing pause time corresponds to decreasing node mobility. As nodes become stable possibility of route breakage is reduced and chances of successful transmission of data is increased. In AODV protocol route is formed on demand. Due to this probability of data transmission is increased and throughput increases to some extent. It is observed that mobility of nodes does not affect the throughput of ad hoc network significantly. Throughput measure approximately 46 to $49 \mathrm{kbps}$ for the pause time varying between 0 and $100 \mathrm{~ms}$.

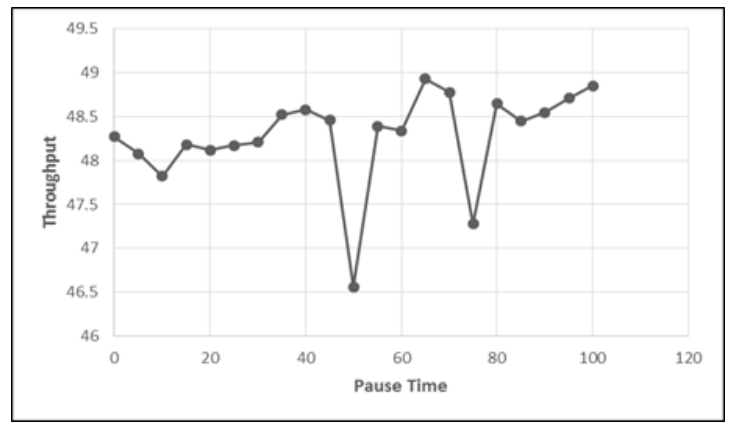

Fig.4. Pause Time vs. Throughput

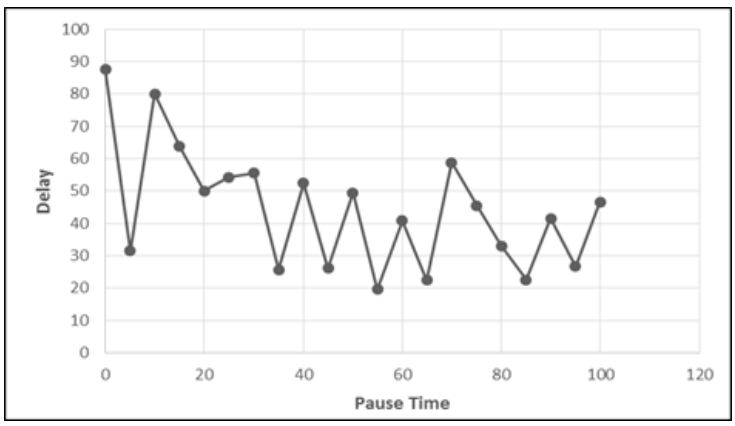

Fig.5. Pause Time vs. Delay

\subsection{Pause Time vs. Delay}

The graphical representation for pause time vs. delay is shown in fig 5. It is observed that delay is variable with changing pause time. In AODV protocol routes are not stored prior. When any node wants to transfer data then route is generated accordingly. The time required to create a route and transmit data depends on the availability of destination node and traffic in the network. Due to this delay is variable in the ad hoc network. As pause time is increasing nodes are becoming more stable which helps in reducing delay to some extent in the ad hoc network. 


\subsection{Pause Time vs. PDF}

As pause time increases from 0 to $100 \mathrm{~ms}$ packet delivery factor (PDF) is approximately constant as shown in fig.6. Packet delivery factor means the total number of packet received with respect to packet sent by the sources. In AODV protocol, two factors are responsible for increasing PDF. The first factor is increase in pause time reduces node mobility which ultimately reduces route breakage. Another factor is routes are created on demand which increases the possibility of data delivery. It is also observed that sometimes packet delivery fraction is decreasing below $90 \%$. This is due to increase in delay and routing load. In AODV protocol routes are formed on demand but if nodes are not available to transfer the data then route settling time increases which increase the delay in the network.

\subsection{Pause Time vs. Routing Load}

Graphical analysis of pause time vs. routing load is shown in fig.7. As pause time is increasing routing load is decreasing. Actually, in AODV protocol, a number of control packets required are more as compared to DSDV protocol. However when pause time increases, network become more stable. Hence, route breakage reduces. No control packets are required to broadcast broken link. Whenever there is a need to transfer the data from source to destination then the only route is created. Hence, no unnecessary control messages are required. This decreases routing load with increasing pause time.

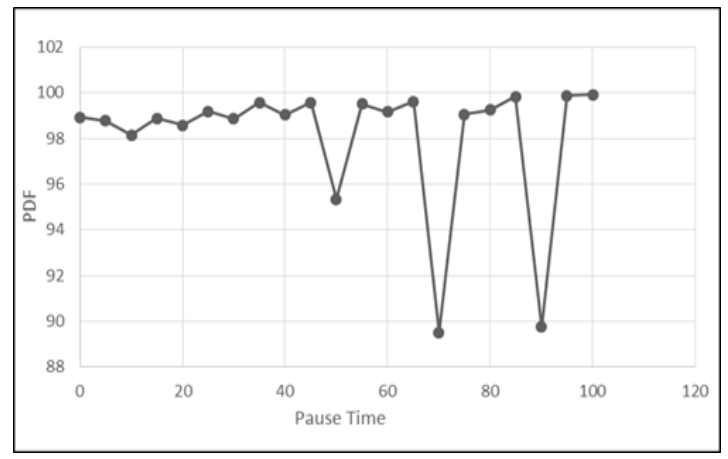

Fig.6. Pause Time vs. PDF

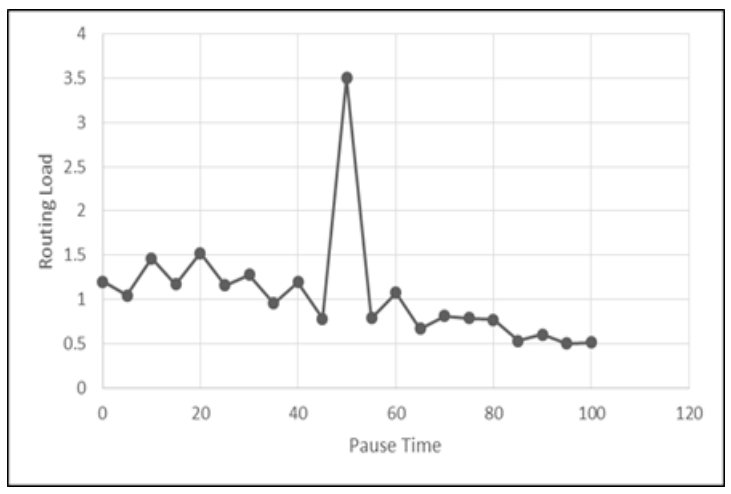

Fig.7. Pause Time vs. Routing Load 


\section{Conclusions}

The performance analysis of existing routing protocol is necessary to study efficiency of an ad hoc network. The AODV protocol is important routing protocol used in Ad hoc network. It creates a route on demand when source node initiates the route request. In this paper the performance of ad hoc network is scrutinized considering various performance parameters like throughput, delay, routing load and packet delivery fraction. In this experiment different self-created scenario file and CBR files are used for simulation through TCL file. Performance of AODV protocol is analyzed against pause time varying in the range 0 to $100 \mathrm{~ms}$ and rest of the network scenario parameters are kept constant. Pause time term correspond to the mobility of nodes. It is observed that as pause time is increasing throughput and PDF is increasing up to some extent however delay, routing load is decreasing. This is because as pause time increases network become stable hence probability of route failure is less. It means when nodes become stable protocol performance is increasing and thus network performance increases. However, practically mobility factor is unpredictable in ad hoc network. So it is essential to regulate mobility of ad hoc network to maintain the performance of the network.

\section{References}

[1] "Dmitri D. Perkins, Herman D. Hughes, and Charles B. Owen", Factors Affecting the Performance of Ad Hoc Networks", 0-7803-7400-2/02/@ 2002 IEEE.

[2] Dr. Sanjay Sharma, Pushpinder Singh Patheja," Improving AODV Routing Protocol with Priority and Power Efficiency in Mobile Ad hoc WiMAX Network “,International Journal of Computer Technology and Electronics Engineering (IJCTEE), vol2, issue1, ISSN 2249-6343.

[3] Dr.Umadevi Chezhiyan, "Measurment Based Analysis of Reactive Protocols in MANET", International Journal of Wired Communications Vol.1, Issue 2, April, 2013.

[4] Jitendra Moond, Dharm Singh, Naveen Choudhary," Advancements in AODV Routing Protocol - A Review" National Conference on Recent advances in Wireless Communication and Artificial Intelligence (RAWCAI-2014), International Journal of Computer Applications (0975 - 8887).

[5] Prashant Kumar Maurya , Gaurav Sharma, Vaishali Sahu , Ashish Roberts , Mahendra Srivastava,"An Overview of AODV Routing Protocol", International Journal of Modern Engineering Research (IJMER) Vol.2, Issue.3, May-June 2012 pp-728-732 ISSN: 2249-6645.

[6] S H Manjula, C N Abhilash, Shaila K, K R Venugopal, L M Patnai ,"Performance of AODV Routing Protocol using Group and Entity Mobility Models in Wireless Sensor Networks"

[7] Sheng Liu, Yang Yang, Weixing Wang ,"Research of AODV Routing Protocol for Ad Hoc Networks1" 2013 AASRI Conference on Parallel and Distributed Computing and Systems, Available online at www.sciencedirect.com

[8] Siddharth Singh, Dr.Naveen Hemrajani, "Performance Evaluation of AODV Routing Protocol in Wireless Sensor Networks with the Constraints of varying terrain areas by varying pause time" International Journal of Emerging Trends \& Technology in Computer Science (IJETTCS), Volume 2, Issue 1, January February 2013 ISSN 2278-6856.

[9] Sudhir Goswami, Chetan Agarwal, Anurag Jain, " Location Based Efficient Scheme for Maximizing Routing Capability od AODV Protocol in MANET “,I.J.Wireless and Microwave Tecnologies(IJWMT), vol 3, issue1, May 2015, ISSN 2076-9539.

[10] Zafar Mahmood, Muhammad Awais Nawaz, Dr Mudassar Iqbal, saleem Khan, Ziaul Haq,"Varying Pause Time Effect on AODV,DSR and DSDV Performance"I.J.Wireless and Microwave Tecnologies(IJWMT), vol 5, issue1, March 2015, ISSN 2076-9539. 


\section{Authors' Profiles}

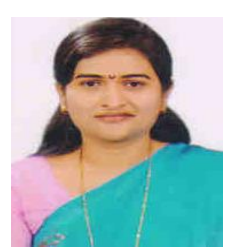

Samiksha Nikam has completed her B.E. in electronics from Shivaji University, Kolhapur. She has completed her M.C.A. from IGNOU, Delhi and MPhil (C.S.) from YCMOU Nashik. She is presently perusing her Ph.D in computer science from Shivaji University, Kolhapur, Maharashtra. Her area of interest is in the field of Ad hoc Network and Fuzzy Logic.

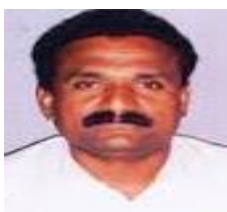

Dr.Bharat Jadhav has obtained his Ph.D degree in electronics from Shivaji University, Kolhapur. Currently he is the Head of Computer Science and Electronics department of Y.C Institute of Sceinces, Satara, Maharashtra. His area of interest is in the field of Network and Fuzzy Logic.

How to cite this paper: Samiksha Nikam, B.T. Jadhav,"Analysis of AODV Protocol against Pause Time Using NS2.34", International Journal of Wireless and Microwave Technologies(IJWMT), Vol.6, No.6, pp.6371, 2016.DOI: 10.5815/ijwmt.2016.06.07 